\title{
Communication Networking Schemes for Wide Area Electric Vehicle Energy Service Network
}

\author{
Dequan Gao ${ }^{1,2}$, Jinping Cao ${ }^{3}$, Yiying Zhang ${ }^{1,2}$, Xiuli Wang ${ }^{4}$ \\ ${ }^{1}$ Beijing University of Posts and Telecommunications, Beijing 100876, China \\ ${ }^{2}$ State Grid Information \& Telecommunication Company Ltd., Beijing 100761, China \\ ${ }^{3}$ China Electric Power Research Institute, Beijing 100192, China \\ ${ }^{4}$ Information Communication Branch of Beijing Electric Power Company, Beijing 100031, China \\ Email: gaodeq@whu.edu.cn
}

Received March, 2013

\begin{abstract}
Electric vehicles (EVs) are an emerging type of mobile intelligent power consumption devices in Smart Grid as new green transport tools. In order to provide a powerful automation and intelligence support for wide area electric vehicles energy service network, we analyze the network infrastructure and communications demands of various terminals, devices and monitoring systems distributed in wide area electric vehicle energy service network. According to interactive user services scenarios and energy operations intelligent monitoring, we propose multimode communication integration architecture for wide area electric vehicle energy service network by means of the fusion of the Internet of Things (IoT) technology. Then, we design different networking schemes in access networks and backbone transmission networks meeting multi-scene and multi-operation interaction requirements. The networking schemes will provide efficient technical support to implement intelligent, cross-regional, interactive energy services for electric vehicle users.
\end{abstract}

Keywords: Electric Vehicle; Wide-area Energy Service Network; Communication Networking; Internet of Things

\section{Introduction}

Many developed countries and megacities are undertaking serious environmental pollution and great oil shortage challenges. So world automobile industries are turning to develop clean energy vehicles with huge investment. Now, many people have realized that future trends of automobile industry are green, energy-saving and intelligent. Electric vehicles (EVs) are seen as one of the most promising means to improve the near-term sustainability of the transportation and stationary energy sectors [1]. Electric vehicles have improved their performance and made suitable for commercial and domestic use during the last decades [2]. Electric vehicles will be prominently taken as the mobile infrastructures of electrified transportation mode. Electric vehicles development has been as one of new energy national strategies in China. More importantly, electric vehicle is an emerging type of mobile intelligent power consumption device and energy storage terminal for Smart Grid. With the large-scale population of electric vehicles, electric vehicles can be conventionally charged to play the role of the valley filling in the power grid while its load is low. Thus, they will improve the comprehensive utilization efficiency of power generation equipment, and achieve the effect of energy saving and emission reduction. Inevitably, to construct wide-area energy service network for electric vehicles is the core premise of their large promotion. According to the $12^{\text {th }}$ Five-Year special plan on electric vehicle technology development in China, a networked power supply system, which includes 400,000 charging piles and 2000 charging-swap stations, will be built in above 20 demonstration cities and their surrounding areas to meet the energy supply requirements of largescale commercial demonstration of electric vehicles until about 2015.

The domain of electric vehicles opens new business perspectives and opportunities [3]. Electric vehicle energy services businesses mainly cover battery charging, battery swap, battery discharging, battery distribution, electric energy metering and billing, fund settlement, scheduling monitoring, etc. the basic operations functions are devices running IntelliSense, real-time monitoring and warning of battery status, vehicle status information acquisition, charging-swap path intelligent navigation, battery lifecycle management, centralized monitoring of equipment, optimal allocation of resources, etc. The distributed smart charging-swap information in energy service network should be measured, interacted, shared and controlled, which necessarily relies on rapid and reliable information communication system. 
Next generation electric vehicles will radically change the design paradigms in the automotive network domain [4]. However, information communications network supporting wide-area electric vehicle information acquisition and key charging-swap service operations and monitoring, has not met the practical, interactive requirements. These problems have made intelligent interaction more difficult between the power grid and users, and led to be unable to provide conveniently, efficient and safe services for electric vehicles users. Therefore, reliable information communication technology (ICT) should be developed to support information perception, aggregation, interaction, and highly automated, intelligent charging-swap services. This paper focuses on the objectives of providing efficient and interactive electric vehicles smart energy services. Some exploratory researches involve wide-area electric vehicles energy services network communications networking architecture. In this paper, we synthetically apply the Internet of Things (IoT) technology and power communication to improve intelligent perception of electric vehicles energy information and services integration level between electric vehicles and power grid.

\section{EV Energy Service Network Facilities}

Electric vehicle energy service network is indispensable infrastructure for large-scale commercialization of electric vehicles. Many Chinese cities have released preferential policies and invested massive funds to promote the construction of electric vehicle energy service facilities. Generally, electric vehicle energy service network facilities are composed of centralized charging stations, battery swap stations, battery distribution stations, AC charging pile, operation monitoring center, etc. They will guarantee reliable energy supply for electric vehicles in a wide area.

Centralized charging stations can provide electric vehicle battery charging and battery distribution stations. Typically, a centralized charging station has the capabilities of charging, discharging, maintenance and detection of large amount of standardized battery packs.

Battery swap stations are power battery supply spots by exchanging batteries for electric vehicle users. In a battery swap station; there are power supply area, charging area, battery replacement area, battery inspection and maintenance area, control room, parking lot, etc. Battery distribution stations provide battery replacement and logistics services for battery packs distribution without charging function. AC charging piles are slow charging devices for electric vehicles. The functions of a charging pile involve timing, power energy measurement, costing calculation. Operation service centers are responsible for monitoring and management of regional electric vehicle energy service network operators.

\section{Communication System Architecture of Wide Area EV Energy Service Network}

Shifting bulk loads and demand response programs require communication between consumers and producers of energy and will be more widely enabled with the expansion of grid communication networks across the country [5]. The communication system of electric vehicle energy service network will achieve the interconnection and interoperability of all kinds of terminal, electric vehicle and operations management system. The communication system can support orderly, seamless cross-regional communication services in the cities, provinces, inter-city and inter-provincial areas.

\subsection{Communication System Demands}

The characteristics of the communication system are flexible networking, multi-mode access, and massive access points. We analyze its demands from the view of security, real-time and bandwidth. As an important part of intelligent distribution and utilization networks, it is linked with the power grid enterprise businesses such as energy metering, tariff settlement, electricity management. So power grid must be safe and stable when a large number of wide-area distributed charge-swap devices are linked into it. Therefore, security requirements of widearea communication network are rather higher for operation service center.

All business surveillance, operations monitoring, video monitoring, battery monitoring, power supply monitoring, logistics distribution information of centralized charging stations, battery-swap stations, battery distribution station, and service management center must be real-time uploaded, so the real-time requirements of communication performance are highly efficient. Moreover, electric vehicles (including logistics vehicles) will upload vehicle conditions, battery status information and receive valueadded services and scheduling information, relatively, their real-time communication requirements are also higher.

Electric power information acquisition system, operation management system, station-level monitoring system need be deployed in centralized charging stations and battery swap stations. Their communication bandwidth is expected to reach MB level. The communication bandwidth of charge piles and electric vehicles is approximate $30 \mathrm{kbps}$.

\subsection{Communication System Architecture}

At present, there are some technical problems restricting the large-scale commercial development of electric vehicles in China. For example, the existing communication networks cannot cover both urban and rural areas, cross area to support complex electric vehicles services. Thus, 
we must integrate power communication network, the public network (GPRS/3G/4G), cable network, wireless sensor networks (WSN), and other communications technology to establish top-level communication system architecture of the electric vehicle energy services network. The communication system will smart, effective interaction between electric vehicles, battery packs, charging-swap facilities and power grid, and seamless mobile information services.

According to the above demand analysis of electric vehicle energy network communication system, we propose three-layer communication network architecture supporting dynamic access and switch, as shown in Figure 1. The network architecture will establish crossregional information transmission covering headquarters operation service center, provincial operation service centers, municipal operation service centers and station monitoring center.

First-level network is the backbone telecommunication network linking operation service headquarters and subordinate operation service centers. First-level network adopts power communication private network. However, for the construction of power communication private network relies on power transmission line path, it actually hasn't covered some expressways, provincial highways. Power companies have built current communications network involving optical fiber cable, microwave, Power Line Carrier (PLC), satellite, which have fully covered headquarters, provincial and municipal companies. Therefore, the communication system of electric vehicle en- ergy service network can further extend current enterprise network to solve the wide-area coverage problem.

Second-level network is the transmission network linking subordinate operation service centers and station level facilities. Second-level network mainly adopts provincial power communication private network. If the private network isn't available, the public communication network can be rented. Here, Access networks can apply WSN, Optical Fiber Composite Low-voltage Cable (OPLC), 3G/GPRS/4G/TD_LTE to implement information exchange between charging-swap devices, vehicle automatic identification equipment and monitoring equipment, and information interaction between inner equipment inside charging-swap stations, electric vehicles and battery packs, and information access of geographically distributed charging piles. On the whole, the communication network coverage is small for low-voltage side distribution network. We can adopt wired communications (optical fiber communications, etc.), IoT communication technologies and public wireless communications (GPRS/3G/4G) for different scenarios to aggregate a variety of perception information, for example, battery status information, identity information, electric vehicle status information, location information, the smart electric card identity information, charging-swap environment information. The aggregated information will be uploaded to the Second-level network layer through the IoT aggregation gateway. Meanwhile, charging-swap devices and various intelligent terminals also receive control instructions.

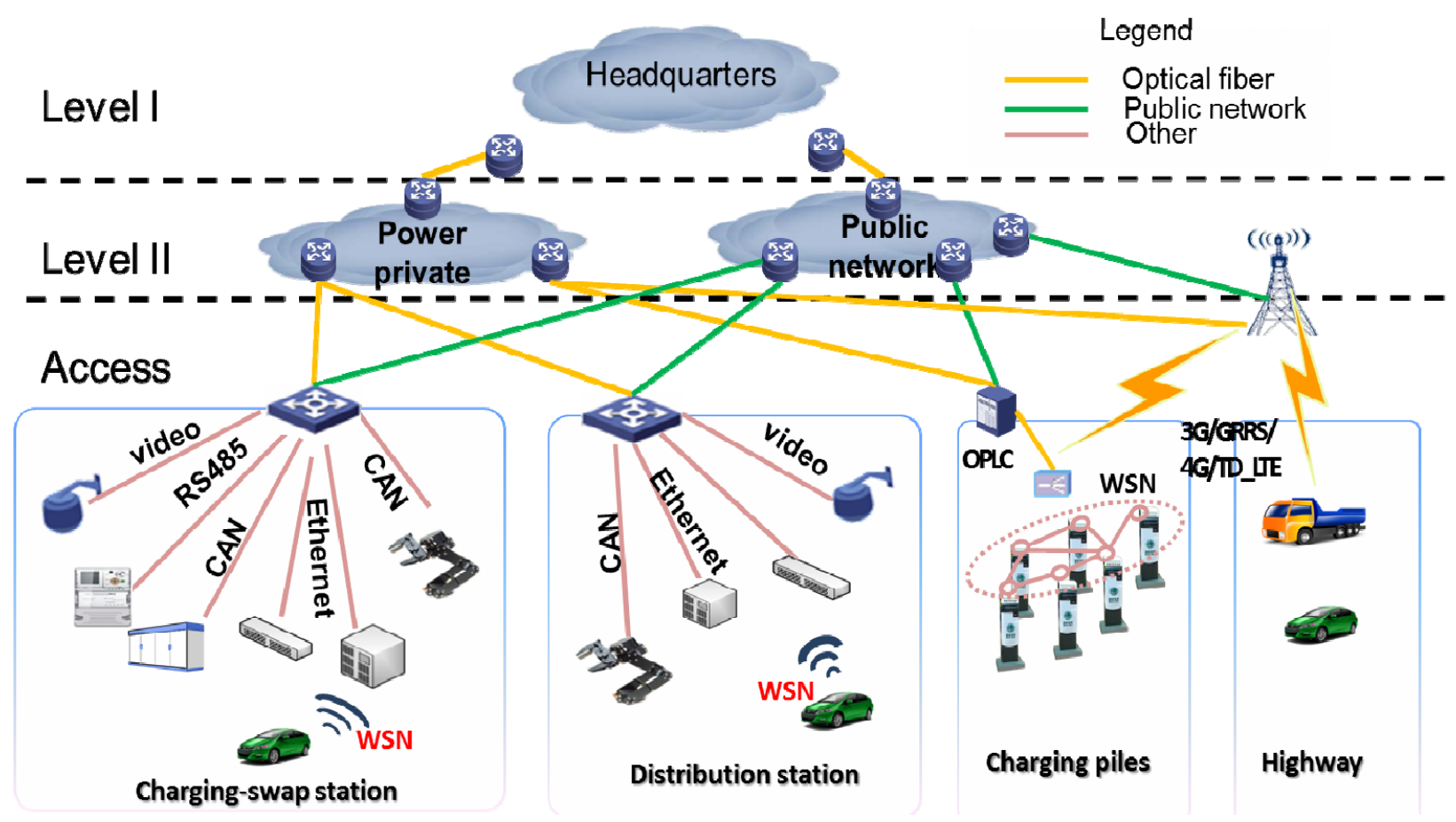

Figure 1. Three-layer communication network architecture. 


\section{Networking Schemes of Access Networks}

Now the backbone telecommunication transmission networks and data communication network built by power grid companies can basically meet communication business demands of the First-level and Second-level network in the energy service network architecture. Here, we will mainly focus on designing networking schemes of access network layer and take low-voltage power communication network as main basic of access network. We will use self-built fiber network, electricity wireless broadband network, IoT technology together with renting public network to solve heterogeneous interconnection and collaborative communication problems for power wireless broadband network, mobile communication network, and WSN applied in different application scenarios. a particular IoT service can be applied in order to optimize application development and speed up application implementation [6]. Monitoring and automation applications will be central for IoT and rely on eventing and group communication [7]. These networking schemes will realize seamless network coverage for centralized charging stations, battery swap station, battery distribution stations, charging pile and electric vehicles, and network access within station sites.

\subsection{Networking Scheme of Centralized Charging Stations}

The communication network within a centralized charging station will connect with access network through the access gateway nodes. The operations, power utilization and monitoring information on the station LAN will be transmitted to provincial service center, and share data with electric vehicle operation management system. The networking structure inside a centralized charging station is shown in Figure 2. The following is networking description.

1) Switches will be core nodes interconnection of equipment inside centralized charging stations to support station-level operations. A workstation connects core switches via Ethernet interfaces.

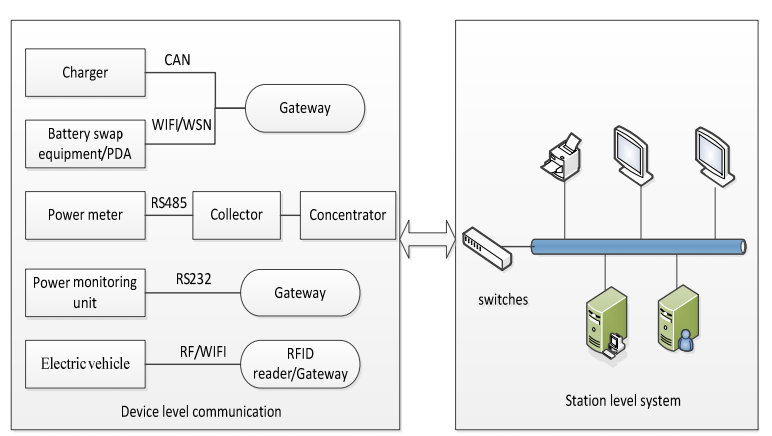

Figure 2. Networking scheme inside centralized charging station.
2) Charging devices access IoT communication gateway via industrial Ethernet or CAN bus, by which they upload battery information and charger information to the monitoring system, and also receive command instructions.

3) A power supply monitoring unit accesses IoT communications gateway via RS232 interface. A collector uses RS485 interface to communicate with electric energy meters and collect real-time consumption information.

4) Video terminals send surveillance data to station operation monitoring system via video cables.

5) Logistics vehicles can use micro-power wireless network to automatically communicate with vehicle identification readers inside a station.

In order to ensure station-level information network security, local centralized charging station business systems are logically isolated via Virtual LAN (VLAN) technology.

\subsection{Networking Scheme of Battery Swap Stations}

According to the practical communication requirements in a battery swap station, the swap devices usually use wireless communication technologies to link station-level monitoring system, for example, WIFI, ZigBee. The operations, power consumption and monitoring information of a battery swap station will be sent to regional operation management system via uplink network by renting special public communication network.

In urban areas, a battery swap station will connect the nearest $10 \mathrm{kV}$ switching substation, 35/110/220 kV substation with fiber optic cable resources. While in intercity highways or highway service areas, a battery swap station will connect the nearest 35/110/220 kV substation with fiber optic cable resources. The uplink communications network fiber optic cable can be laid along with power lines.

\subsection{Networking Scheme of Charging Piles}

Charging piles usually have CAN bus, PLC and RS485 communication interfaces, by which charging status information can be sent to centralized monitoring system. Charging piles can Real-time communicate with battery management system (BMS) to get battery type, single voltage, State-of-Charge (SOC), temperature and warning information.

The uplink networks of charge piles have two access ways: self-built power private networks and rented public networks.

The operations information, power consumption information and monitoring information can be uploaded via LAN deployed in $10 \mathrm{kV}$ distribution room, switching 
cabinet, box-type substation. Also, they can use embedded wireless communication modules to connect public network.

\subsection{Networking Scheme between Charging-swap Facilities and Operation Service Centers}

The key communication requirements between chargingswap facilities and the operation service centers of energy network are wide communication range, rich data semantic content and various bandwidth applications, so we must flexibly use multiple communication technologies for different application scenarios. Power optical fiber, PLC, GPRS and other communication technologies can be mixedly used to communicate with operation service centers for charging-swap stations and distributed charging piles. For power fiber cables only rely on electric power communication systems resources, they can avoid technical conflict on frequency resources, routing coordination and electromagnetic compatibility with other users. PLC technology is unique communication way for power industry. The PLC scheme doesn't need to newly build communications infrastructure for it uses power line as communication media for data transmission and information exchange. Station-level management systems can real-time interact with electric vehicles energy service management system in operation service center, electric energy data acquire system and 95598 call center through power fiber-optic network.

\subsection{Networking Scheme of Electric Vehicles}

The main external interaction objects of electric vehicle are operation service centers, charging-swap stations and charging devices. For mobility of electric vehicles, their driving range, spatial location and speed are random. Obviously, different communication technologies will be applied inside electric vehicles or while they are moving in urban areas or on inter-city highways. The networking schemes of electric vehicles are described in Figure 3.

Interactive services of electric vehicles in different scenarios will adopt different communication modes. Careful monitoring and control of energy flows allows for minimum investment with respect to cost, weight and

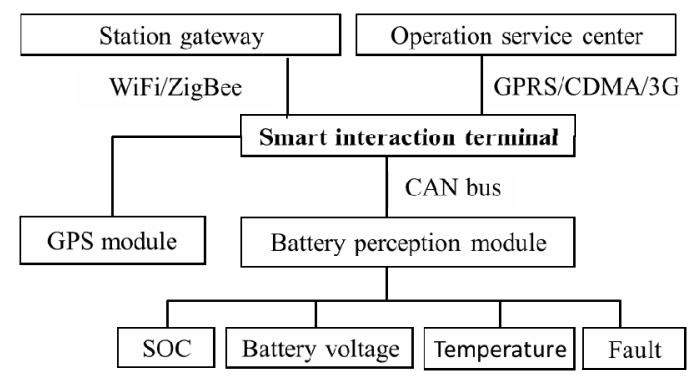

Figure 3. Networking scheme of electric vehicles. volume [8]. Users can get real-time battery status data via vehicle CAN bus interface interacting with BMS while driving. The application of bus technology with CAN as the representative in vehicles not only reduces the har-ness of the car but also increases the reliability of the car [9]. IoT based smart interactive terminals can be embedded into electric vehicles to realize perception information transmission among different interior parts. Smart interactive terminals integrate battery energy sensor, temperature sensor, identification chip, communication unit, GPS module, navigation software to realize battery packs identification, power information collection, speech warning and map navigation, etc.

Electric vehicles can use WIFI, micro-power wireless network to interact with smart terminals via Iot gateways set in charging-swap stations. Furthermore, they generally interact with charging devices via CAN interface.

Users often adopt renting public wireless communication network to interact with service centers via GPRS/ 3G/4G outside charging-swap stations. In the future, the new generation power wireless broadband private network and White Space (WS) technology-based low-frequency wireless communication can be used to achieve regionscale coverage data transmission. In general, wireless mobile communication network can be used as the primary networking means, the roadside big WiFi AP networks and TD-LTE based new power wireless broadband communications serve effective supplement means.

\subsection{Communication Protocols and Interfaces Security}

The main communication interfaces of electric vehicles energy service Network are data communication interface of charging-swap devices, information interoperation interface of operation monitoring center and BMS interface of electric vehicles. In order to establish universal communication protocol, we must find out the common characteristics of electric vehicle charging demands and charging-swap device functions from too many manufacturers. However, the communication protocol for electric vehicles hasn't been already unified for the BMS interfaces from different manufacturers are special. To actually ensure real-time data upload of monitor electrical equipment operating condition, switch status and fault alarm in charging-swap facilities, the modified IEC 60870-5-104 protocol can be widely used.

In order to ensure wired communications security inside charging-swap stations, the monitoring systems of charging-swap and power supply management must only access the internal information network through communication front-end processor. Station-level security and protection subsystems access upwards the network to implement data transmission of video stream. Moreover, the devices inside a station must firstly be requested to 
traverse firewalls for ensuring network security if they will access to the internal information network. Under some special conditions, public wireless networks may be used in charging-swap stations if there are no wired power network links. In the above case, the communication front-end processor must be equipped with a radio signal transceiver and a SIM card. Meanwhile, The SIM card should have a static IP address. Thus, the equipment in a station will access the internal information network via public wireless communication network according to the APN (Access Point Name) special line of a station system

\section{Conclusions}

The reliable information collection, transmission, control and management of electric vehicles and charging-swap facilities are some important links to effectively supervise energy service procedure. In order to adapt mobility, diversity and universality requirements of electric vehicle energy services, we make deep demand analysis on the networking infrastructure and communications demands of diverse terminals, devices and monitoring systems distributed in wide area electric vehicle energy service network. According to energy service scenarios for electric vehicle and systematic requirements of intelligent operation monitoring, we propose multimode communication architecture for supporting wide area electric vehicle energy service network with the fusion of IoT technology. The architecture involves hybrid networking mode widely covering expressways, national highways, provincial highways, charging-swap facilities to implement seamless coverage and dynamic access of energy service information. We integrate Multimode communication technologies to design interactive networking schemes for EVs multi-business energy services. The networking schemes will partly solve some basic problems of perception data aggregation and information communication support for cross-regional energy services, and provide some technical means for friendly information interaction.

Electric vehicle energy service infrastructures will have rapid development in next decades in China. In future, it is urgently necessary to innovatively use IoT technology to build a demonstration platform of data acquisition and information management for electric vehicles energy service network. Moreover, the research on information interaction methods between electric vehicle and charging-swap networks should be further done. Wireless smart sensors, perception tags, GPS and other IntelliSense technologies need be integrated to fully collect data in physical world for reliably solving the problem of the electric vehicle performance assessment, fault diagnosis, safety pre-warning and error risks analysis.

\section{Acknowledgements}

This work is supported by National High-tech R\&D Program of China (863 Program) (No. 2011AA05A116, 2012AA050804) and National Program on Key Basic Research Project of China (973 Program) (No. 2011CB302900).

\section{REFERENCES}

[1] C. Quinn, D. Zimmerle and T. H. Bradley, "The Effect of Communication Architecture on the Availability, Reliability, and Economics of Plug-in Hybrid Electric Vehicle-to-grid Ancillary Services,” Journal of Power Sources, Vol. 195, No. 5, 2010, pp. 1500-1509.

doi:10.1016/j.jpowsour.2009.08.075

[2] J. Moreno, M. E. Ortúzar and J. W. Dixon, "Energy-management System for a Hybrid Electric vehicle, Using Ultracapacitors and Neural Networks," IEEE Transactions on Industrial Electronics, Vol. 53, No. 2, 2006, pp. 614-629. doi:10.1109/TIE.2006.870880

[3] N. Masuch, M. Lutzenberger, S. Ahrndt, A. Heßler and S. Albayrak. "A Context-aware Mobile Accessible Electric Vehicle Management System," Proceedings of the Federated Conference on Computer Science and Information Systems, Szczecin, 18-21 September 2011, pp. 305-312.

[4] M. Lukasiewycz, S. Chakraborty and P. Milbredt, "FlexRay Switch Scheduling-A Networking Concept for Electric Vehicles,” Design, Automation \& Test in Europe Conference \& Exhibition (DATE), Grenoble, 14-18 March 2011, pp. 1-6.

[5] T. Markel, M. Kuss and P. Denholm, "Communication and Control of Electric Drive Vehicles Supporting Renewables," IEEE Vehicle Power and Propulsion Systems Conference, Dearborn, 7-10 September 2009, pp. 27-34.

[6] M. Gigli and S. Koo, "Internet of Things: Services and Applications Categorization," Advances in Internet of Things, Vol. 1, No. 2, 2011, pp. 27-31.

doi:10.4236/ait.2011.12004

[7] M. Kovatsch, “A User-Centered Application Layer for the Internet of Things”, ACM SenSys'11, Seattle, 1-4 November, 2011.

[8] E. Meissner and G. Richter, "Battery Monitoring and Electrical Energy Management Precondition for future vehicle electric power systems", Journal of Power Sources, Vol. 116, No. 1, 2003, pp. 79-98.

doi:10.1016/S0378-7753(02)00713-9

[9] Q. Q. Zhang, Y. Wang and T. M. Yin, "Design of the Control System about Central Signals in Electric Vehicle,” Journal of Electromagnetic Analysis \& Applications, Vol. 2, No. 3, 2010, pp. 189-194.

doi:10.4236/jemaa.2010.23027 